\title{
Associations between CT pulmonary opacity score on admission and clinical characteristics and outcomes in patients with COVID-19
}

\author{
Huanyuan Luo ${ }^{1}$ (1) Yuancheng Wang ${ }^{2} \cdot$ Songqiao Liu $^{3} \cdot$ Ruoling Chen $^{4} \cdot$ Tao Chen $^{1} \cdot$ Yi Yang $^{3} \cdot$ Duolao Wang $^{1}$. \\ Shenghong Ju
}

Received: 16 February 2021 / Accepted: 10 June 2021 / Published online: 30 June 2021

(c) Società Italiana di Medicina Interna (SIMI) 2021

\begin{abstract}
This study investigated associations between chest computed tomography (CT) pulmonary opacity score on admission and clinical features and outcomes in COVID-19 patients. The retrospective multi-center cohort study included 496 COVID-19 patients in Jiangsu province, China diagnosed as of March 15, 2020. Patients were divided into four groups based on the quartile of pulmonary opacity score: $\leq 5 \%, 6-20 \%, 21-40 \%$ and $41 \%+$. CT pulmonary opacity score was independently associated with age, single onset, fever, cough, peripheral capillary oxygen saturation, lymphocyte count, platelet count, albumin level, C-reactive protein (CRP) level and fibrinogen level on admission. Patients with score $\geq 41 \%$ had a dramatic increased risk of severe or critical illness [odds ratio (OR), 15.58, 95\% confidence interval (CI) 3.82-63.53), intensive care unit (ICU)] admission (OR, 6.26, 95\% CI 2.15-18.23), respiratory failure (OR, 19.49, 95\% CI 4.55-83.40), and a prolonged hospital stay (coefficient, $2.59,95 \%$ CI $0.46-4.72$ ) compared to those with score $\leq 5 \%$. CT pulmonary opacity score on admission, especially when $\geq 41 \%$, was closely related to some clinical characteristics and was an independent predictor of disease severity, ICU admission, respiratory failure and long hospital stay in patients with COVID-19.
\end{abstract}

Keywords COVID- $19 \cdot$ Coronavirus $\cdot 2019-\mathrm{nCoV} \cdot \mathrm{CT} \cdot$ Pulmonary opacity $\cdot$ Prognosis

\section{Introduction}

Computed tomography (CT) is important in the diagnosis and monitoring of emerging infectious diseases caused by viral infections and manifested mainly in the respiratory

Huanyuan Luo, Yuancheng Wang and Songqiao Liu have contributed equally.

Duolao Wang

Duolao.wang@1stmed.ac.uk

Shenghong Ju jsh0836@hotmail.com

1 Department of Clinical Sciences, Liverpool School of Tropical Medicine, Liverpool L3 5QA, UK

2 Department of Radiology, Zhongda Hospital, School of Medicine, Southeast University, Nanjing 210009, China

3 Jiangsu Provincial Key Laboratory of Critical Care Medicine, Department of Critical Care Medicine, Zhongda Hospital, School of Medicine, Southeast University, Nanjing 210009, China

4 Faculty of Education, Health and Wellbeing, University of Wolverhampton, Wolverhampton WV1 1LY, UK tract [such as H1N1 and severe acute respiratory syndrome (SARS)] [1, 2]. COVID-19 is a clinical infectious disease resulting in bilateral pneumonia and rapid deterioration of lung function [3]. Although real-time reverse transcriptasepolymerase chain reaction assay (RT-PCR) is the standard diagnostic method for COVID-19, chest CT plays an important supplementary role in the diagnosis [4] with a high sensitivity but a lower specificity [5]. CT findings of COVID-19 are mainly patchy glass opacity in the peripheral area $[6,7]$. Previous studies have shown that the abnormal imaging of COVID-19 patients was correlated with main clinical symptoms [8], demographic, epidemiologic, clinical, laboratory characteristics, treatments, severe/critical pneumonia [9], maximal respiratory severity score [10], intensive care unit (ICU) admission [11], and length of hospitalization [12].

This large multi-center cohort study aims to systematically investigate associations between CT pulmonary opacity score on admission and clinical features and outcomes in COVID-19 patients. 


\section{Materials and methods}

\section{Study population}

Inclusion criteria were as of March 15, 2020, all patients $(N=631)$ diagnosed with COVID-19 in all 24 hospitals designated for COVID-19 treatment in Jiangsu province, China according to the "Diagnosis and Treatment Protocol for Novel Coronavirus Pneumonia (Trial Version 7)" released by the National Health Commission and National Administration of Traditional Chinese Medicine of China [13]. Exclusion criteria: Patient with no available medical records $(N=6)$ or CT pulmonary opacity score $(N=129$, did not take CT but X-ray examination). The study ultimately included 496 cases.

\section{Data collection and definition of variables}

Data were extracted from medical records provided by the Data Centre of Jiangsu Provincial Health Commission of China. Variables analyzed were demographic features (age, sex), epidemiological features [type of disease onset (single onset or clustering onset)], clinical features on admission (initial symptoms, medical history, vital signs), radiologic features on admission (CT pulmonary opacity score), laboratory features on admission (hematology, organ function, inflammatory factors, coagulation function indicators), clinical management during hospitalization (supportive treatments and medical drugs), and clinical outcomes (disease severity, ICU admission, respiratory failure, length of hospital stay). A clustering onset was defined as the occurrence of two or more confirmed COVID-19 cases in the same cluster/group within 14 days, such as family and hospital. Other cases were classified as single onset. Two radiologists (YW and SJ) with more than 5 years of working experience in chest imaging performed visual evaluation of CT images and the agreement was reached through consultation if discrepancies of pulmonary opacity score occurred. Pulmonary opacity score was defined as the percentage of pulmonary opacity [ground-glass opacities (GGO) or consolidation] area relative to the entire lung on $\mathrm{CT}$ images (range $0-100 \%$ ), rounded to the nearest $5 \%$. GGO was defined as an area of hazy increased attenuation, in which vessels and bronchial markings may still be observable. Consolidation was more opaque than GGO, in which such markings were obscured. On CT image, GGO and consolidation looked more gray or hazy compared to the normal dark appearance of the lung. Figure 1 showed an example of CT image for a patient with pulmonary opacity score being $70 \%$.

Disease severity were measured at days 1, 2, 3, 4, 5, 6, 7 and 14 after admission, mortality and hospitalization status were available until March 15, 2020. Asymptomatic infection was defined as the absence of clinical symptoms but a positive RT-PCR result. Mild disease was defined as having mild clinical symptoms without respiratory distress and the absence of imaging manifestations of pneumonia. Moderate disease was the presence of fever, respiratory tract symptoms and imaging manifestations of pneumonia. Severe disease was the presence of at least one of the follows: respiratory distress, respiratory rate $\geq 30$ breaths/minute; peripheral capillary oxygen saturation $\left(\mathrm{SpO}_{2}\right) \leq 93 \%$; or arterial blood oxygen partial pressure $\left(\mathrm{PaO}_{2}\right)$ / fraction of inspired oxygen $\left(\mathrm{FiO}_{2}\right) \leq 300 \mathrm{mmHg}$ $(1 \mathrm{mmHg}=0.133 \mathrm{kPa})$. Critically ill was having respiratory failure requiring mechanical ventilation, shock or combined organ failure requiring ICU monitoring and treatment.

\section{Statistical analysis}

Patients were divided into four groups based on the quartile of baseline CT pulmonary opacity score: $\leq 5 \%, 6-20 \%$, $21-40 \%$ and $41 \%+$. Continuous variables of baseline features and clinical management during hospitalization were reported as mean [standard deviation (SD)] or median [interquartile range (IQR)] by group and compared using ANOVA test or Kruskal-Wallis test depending on their distributions. Binary variables were summarized using frequency and percentage and compared using $\chi^{2} /$ Fisher exact test. To assess the linear trend effect of pulmonary opacity score on baseline features and clinical management, generalized linear model (GLM) was employed with pulmonary opacity score as the only predictor (continuous variable). For GLM analysis of continuous variables, normal distribution and identity link function were used; whereas for GLM analysis of binary variables, binomial distribution and logit link function were used. The analysis of inter-observer agreement for pulmonary opacity scores was conducted using an intraclass correlation coefficient (ICC) calculated from a generalized linear mixed model.

To explore associations between pulmonary opacity score with characteristics on admission, univariate and multivariate linear regression analysis was conducted, with pulmonary opacity score as dependent variable (continuous variable). To assess effects of pulmonary opacity score on clinical outcome, GLMs were performed, in which pulmonary opacity score was treated as a continuous and categorical variable, controlling for baseline characteristics.

Two-tailed $P<0.05$ was considered as statistically significant. The analyses were performed using SAS 9.4 (SAS Institute). 


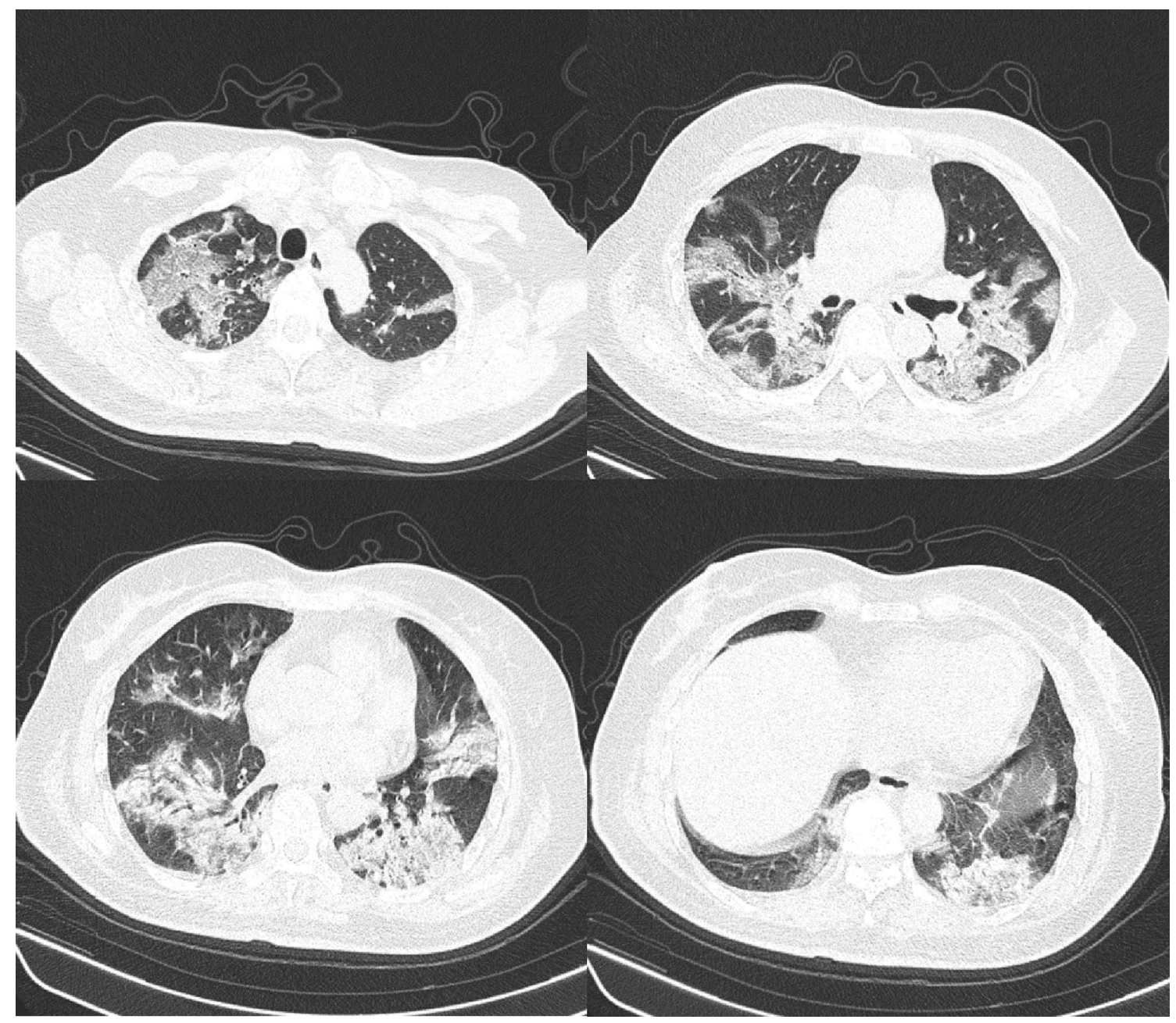

Fig. 1 CT images of a patient with pulmonary opacity score of 70\%. Details: Female, 61 years old. Her CT was performed 1 day after admission. She was diagnosed with respiratory failure on the next day

\section{Results}

Of 496 patients [270 (54.4\%) men, mean age 45.10 (SD 17.13) years], $244(49.2 \%)$ were single onset, 339 (68.3\%) had fever, 279 (56.3\%) had cough, $130(26.2 \%)$ had sputum, $71(14.3 \%)$ had hypertension, 31 (6.3\%) had diabetes, 30 (6.0\%) smoked, mean temperature was $37.09{ }^{\circ} \mathrm{C}$ (SD 0.76), mean heart rate was 87.70 beats/minute (SD 13.42), mean respiratory rate was 19.10 breaths/minute (SD 2.49) and mean peripheral capillary oxygen saturation $\left(\mathrm{SpO}_{2}\right)$ was $97.60 \%$ (SD 2.16) (Table 1). The ICC value for pulmonary opacity score from the two radiologists was 0.93 . With the rise of pulmonary opacity score, age and body temperature statistically significantly increased (trend test) while $\mathrm{SpO}_{2}$ decreased, and the proportion of men and patients who were single onset and who had initial symptoms, hypertension comorbidity and smoking habit increased.

As pulmonary opacity score raised, lymphocyte count, platelet count and albumin level statistically significantly declined, while the level of CRP and fibrinogen elevated (trend test, Table 2). The proportion of patients receiving medical treatment and oxygen support increased with the increase in pulmonary opacity score, except for the rarely used continuous renal replacement therapy and extracorporeal membrane oxygenation, and the commonly used interferon and antivirals (trend test, Table 3 ).

Pulmonary opacity score on admission was independently associated with age, single onset type, presentation of symptoms including fever and cough, vital signs including temperature and $\mathrm{SpO}_{2}$ (Table 4).

Pulmonary opacity score on admission was independently associated with severe or critical illness, ICU admission and respiratory failure (Table 5). Especially, compared to patients with pulmonary opacity score $\leq 5 \%$, those who with score $\geq 41 \%$ had a statistically significant increased odds of severe or critical illness (OR, 15.58, 95\% CI 3.82-63.53, $P=0.0001$ ), ICU admission (OR, $6.26,95 \%$ CI $2.15-18.23, P=0.0008)$ and respiratory 


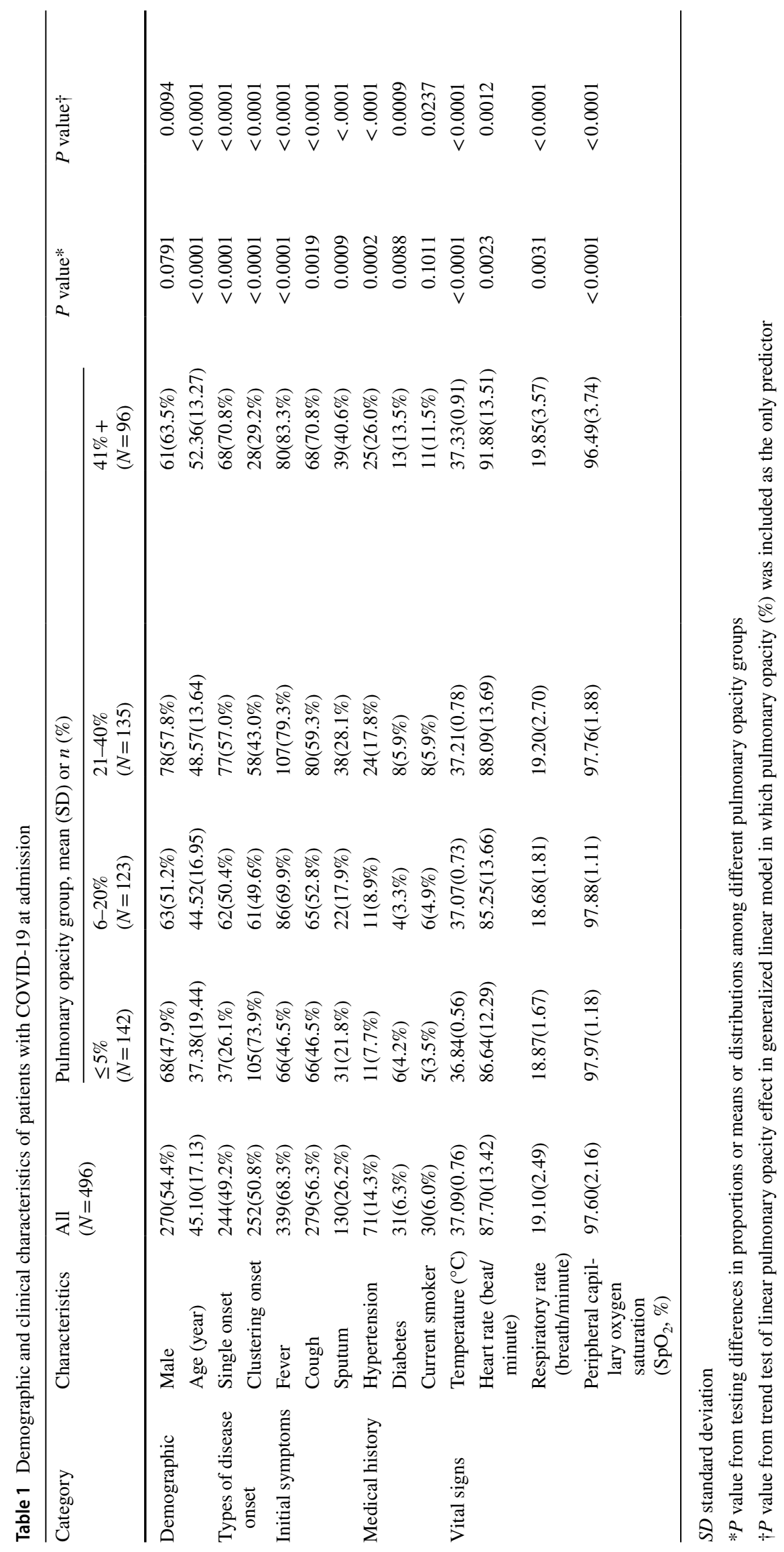


Table 2 Laboratory parameters of patients with COVID-19 at admission

\begin{tabular}{|c|c|c|c|c|c|c|c|c|}
\hline \multirow[t]{2}{*}{ Category } & \multirow[t]{2}{*}{ Parameters } & \multirow{2}{*}{$\begin{array}{l}\text { All } \\
(N=496)\end{array}$} & \multicolumn{4}{|c|}{ Pulmonary opacity group, $N$, median (IQR) } & \multirow[t]{2}{*}{$P$ value* } & \multirow[t]{2}{*}{$P$ value $\dagger$} \\
\hline & & & $\begin{array}{l}\leq 5 \% \\
(N=142)\end{array}$ & $\begin{array}{l}6-20 \% \\
(N=123)\end{array}$ & $\begin{array}{l}21-40 \% \\
(N=135)\end{array}$ & $\begin{array}{l}41 \%+ \\
(N=96)\end{array}$ & & \\
\hline \multirow[t]{5}{*}{ Blood test } & $\begin{array}{l}\text { White } \\
\text { blood } \\
\text { cell } \\
\text { count } \\
\left(10^{9} / \mathrm{L}\right)\end{array}$ & $413,4.8(3.9-6.0)$ & $124,5.2(4.3-6.6)$ & $93,4.6(3.5-5.4)$ & $113,4.4(3.6-5.9)$ & $83,4.9(3.9-6.2)$ & 0.0009 & 0.9967 \\
\hline & $\begin{array}{l}\text { Neutrophil } \\
\left(10^{9} / \mathrm{L}\right)\end{array}$ & $407,2.9(2.1-3.9)$ & $117,3.0(2.3-4.1)$ & $94,2.7(2.0-3.5)$ & $113,2.9(2.1-3.6)$ & $83,3.3(2.5-4.6)$ & 0.0071 & 0.0028 \\
\hline & $\begin{array}{l}\text { Lympho- } \\
\text { cyte } \\
\left(10^{9} / \mathrm{L}\right)\end{array}$ & $405,1.3(0.9-1.7)$ & $117,1.6(1.2-2.1)$ & $93,1.3(1.0-1.6)$ & $112,1.3(0.9-1.6)$ & $83,1.0(0.7-1.3)$ & $<0.0001$ & $<0.0001$ \\
\hline & $\begin{array}{l}\text { Hemo- } \\
\text { globin } \\
\text { (g/L) }\end{array}$ & $\begin{array}{l}410,135.5(123.0- \\
150.0)\end{array}$ & $\begin{array}{l}124,135.5(124.0- \\
149.0)\end{array}$ & $\begin{array}{l}93,135.0(121.0- \\
154.0)\end{array}$ & $\begin{array}{l}111,134.0(118.0- \\
146.0)\end{array}$ & $\begin{array}{l}82,138.0(124.0- \\
154.0)\end{array}$ & 0.4568 & 0.9862 \\
\hline & $\begin{array}{l}\text { Platelet } \\
\left(10^{9} / \mathrm{L}\right)\end{array}$ & $\begin{array}{l}395,182.0(149.0- \\
218.0)\end{array}$ & $\begin{array}{l}123,199.0(160.0- \\
235.0)\end{array}$ & $\begin{array}{l}91,184.0(154.0- \\
211.0)\end{array}$ & $\begin{array}{l}107,175.0(144.0- \\
208.0)\end{array}$ & $\begin{array}{l}74,160.0(123.0- \\
211.0)\end{array}$ & 0.0026 & 0.0002 \\
\hline \multirow[t]{4}{*}{$\begin{array}{l}\text { Organ } \\
\text { function }\end{array}$} & $\begin{array}{l}\text { Alanine } \\
\text { ami- } \\
\text { notrans- } \\
\text { ferase } \\
\text { (U/L) }\end{array}$ & $\begin{array}{l}341,24.9(16.0- \\
37.5)\end{array}$ & $\begin{array}{l}103,22.0(15.0- \\
33.0)\end{array}$ & $\begin{array}{l}82,24.0(14.5- \\
39.3)\end{array}$ & $95,27.0(19.0-38.0)$ & $\begin{array}{l}\quad 61,26.4(19.0- \\
44.0)\end{array}$ & 0.0280 & 0.0003 \\
\hline & $\begin{array}{l}\text { Albumin } \\
\text { (g/L) }\end{array}$ & $\begin{array}{l}396,41.0(37.5- \\
44.3)\end{array}$ & $\begin{array}{l}119,42.0(38.0- \\
46.7)\end{array}$ & $\begin{array}{l}91,41.6(38.8- \\
45.5)\end{array}$ & $\begin{array}{l}107,41.0(37.5- \\
43.7)\end{array}$ & $\begin{array}{l}79,39.8(34.0- \\
42.0)\end{array}$ & 0.0003 & $<0.0001$ \\
\hline & $\begin{array}{l}\text { Total } \\
\text { bilirubin } \\
\text { (umol/L) }\end{array}$ & $381,9.9(6.0-14.3)$ & $114,9.6(5.0-15.6)$ & $85,9.0(6.6-14.1)$ & $106,10.1(6.4-14.9)$ & $76,10.1(5.3-12.9)$ & 0.7289 & 0.8663 \\
\hline & $\begin{array}{r}\text { Creatinine } \\
\text { (umol/L) }\end{array}$ & $\begin{array}{l}392,64.0(51.0- \\
78.4)\end{array}$ & $\begin{array}{l}118,61.0(48.0- \\
78.0)\end{array}$ & $\begin{array}{l}91,65.0(51.0- \\
76.0)\end{array}$ & $\begin{array}{l}103,60.7(47.0- \\
76.0)\end{array}$ & $\begin{array}{l}80,70.5(57.5- \\
86.0)\end{array}$ & 0.0125 & 0.0426 \\
\hline \multirow[t]{2}{*}{$\begin{array}{l}\text { Inflam- } \\
\text { matory } \\
\text { factors }\end{array}$} & $\begin{array}{c}\text { C-reactive } \\
\text { protein } \\
(\mathrm{mg} / \mathrm{L})\end{array}$ & $377,10.0(4.0-25.2)$ & $112,5.1(1.0-10.0)$ & $89,10.0(4.5-16.2)$ & $96,13.4(5.4-26.9)$ & $\begin{array}{l}80,33.6(10.2- \\
67.0)\end{array}$ & $<0.0001$ & $<0.0001$ \\
\hline & $\begin{array}{l}\text { Procal- } \\
\text { citonin } \\
(\mathrm{ng} / \mathrm{mL})\end{array}$ & $355,0.0(0.0-0.2)$ & $100,0.1(0.0-0.2)$ & $85,0.0(0.0-0.1)$ & $102,0.0(0.0-0.1)$ & $68,0.1(0.0-0.4)$ & 0.0007 & 0.1307 \\
\hline \multirow[t]{3}{*}{$\begin{array}{l}\text { Coagula- } \\
\text { tion } \\
\text { function } \\
\text { test }\end{array}$} & $\begin{array}{l}\text { Activated } \\
\text { partial } \\
\text { throm- } \\
\text { boplastin } \\
\text { time (s) }\end{array}$ & $\begin{array}{l}407,33.0(28.0- \\
38.0)\end{array}$ & $\begin{array}{l}121,32.8(27.9- \\
38.0)\end{array}$ & $\begin{array}{l}93,32.9(28.2- \\
37.3)\end{array}$ & $\begin{array}{l}111,32.4(28.0- \\
38.0)\end{array}$ & $\begin{array}{l}82,34.6(28.8- \\
38.3)\end{array}$ & 0.8155 & 0.9293 \\
\hline & $\begin{array}{l}\text { Fibrinogen } \\
(\mathrm{g} / \mathrm{L})\end{array}$ & $393,3.6(2.8-4.4)$ & $115,3.0(2.5-3.7)$ & $90,3.1(2.6-3.8)$ & $109,4.1(3.4-4.4)$ & $79,4.5(3.8-5.5)$ & $<0.0001$ & $<0.0001$ \\
\hline & $\begin{array}{r}\text { D-dimer } \\
(\mathrm{mg} / \mathrm{L})\end{array}$ & $393,0.3(0.2-0.4)$ & $113,0.3(0.2-0.4)$ & $91,0.2(0.2-0.4)$ & $108,0.3(0.2-0.4)$ & $81,0.3(0.2-0.5)$ & 0.5765 & 0.4164 \\
\hline
\end{tabular}

$I Q R$ interquartile range

$* P$ value from testing differences in distributions among different pulmonary opacity groups

$\dagger P$ value from trend test of linear pulmonary opacity effect in generalized linear model in which pulmonary opacity (\%) was included as the only predictor

failure (OR, 19.49, 95\% CI 4.55-83.40, $P<0.0001)$. Pulmonary opacity score was also associated with the length of hospital stay and in the third quartile versus the first quartile, the duration of hospitalization raised significantly (coefficient, 2.59, 95\% CI 0.46-4.72, $P=0.0170$ ).

\section{Discussion}

To our knowledge, this is by far one of the largest studies that systematically assessed associations between CT 
Table 3 Clinical management of patients with COVID-19 during hospital stay

\begin{tabular}{|c|c|c|c|c|c|c|c|c|}
\hline \multirow[t]{2}{*}{ Category } & \multirow[t]{2}{*}{ Clinical management } & \multicolumn{5}{|c|}{ Pulmonary opacity group, $n(\%)$} & \multirow[t]{2}{*}{$P$ value* } & \multirow[t]{2}{*}{$P$ value $\dagger$} \\
\hline & & $\begin{array}{l}\text { All } \\
(N=496)(\%)\end{array}$ & $\begin{array}{l}\leq 5 \% \\
(N=142)(\%)\end{array}$ & $\begin{array}{l}6-20 \% \\
(N=123)(\%)\end{array}$ & $\begin{array}{l}21-40 \% \\
(N=135)(\%)\end{array}$ & $\begin{array}{l}41 \%+ \\
(N=96)(\%)\end{array}$ & & \\
\hline \multirow[t]{9}{*}{ Supportive treatments } & $\begin{array}{l}\text { Inotropic and vaso- } \\
\text { constrictive agents }\end{array}$ & $5(1.0)$ & $0(0.0)$ & $0(0.0)$ & $1(0.7)$ & $4(4.2)$ & 0.0079 & 0.0014 \\
\hline & Nasal cannula & $178(35.9)$ & $34(23.9)$ & $33(26.8)$ & $58(43.0)$ & $53(55.2)$ & $<0.0001$ & $<0.0001$ \\
\hline & Mask & $13(2.6)$ & $0(0.0)$ & $1(0.8)$ & $2(1.5)$ & $10(10.4)$ & $<0.0001$ & $<0.0001$ \\
\hline & $\begin{array}{l}\text { High-flow nasal can- } \\
\text { nula oxygen therapy }\end{array}$ & $20(4.0)$ & $2(1.4)$ & $4(3.3)$ & $2(1.5)$ & $12(12.5)$ & 0.0002 & $<0.0001$ \\
\hline & $\begin{array}{l}\text { Non-invasive ventila- } \\
\text { tion }\end{array}$ & $29(5.8)$ & $2(1.4)$ & $3(2.4)$ & $4(3.0)$ & $20(20.8)$ & $<0.0001$ & $<0.0001$ \\
\hline & $\begin{array}{l}\text { Invasive mechanical } \\
\text { ventilation }\end{array}$ & $5(1.0)$ & $0(0.0)$ & $0(0.0)$ & $1(0.7)$ & $4(4.2)$ & 0.0079 & 0.0007 \\
\hline & Prone position & $15(3.0)$ & $1(0.7)$ & 2(1.6) & $2(1.5)$ & $10(10.4)$ & 0.0003 & $<0.0001$ \\
\hline & $\begin{array}{l}\text { Continuous renal } \\
\text { replacement therapy }\end{array}$ & $1(0.2)$ & $0(0.0)$ & $0(0.0)$ & $0(0.0)$ & $1(1.0)$ & 0.1935 & 0.1339 \\
\hline & $\begin{array}{l}\text { Extracorporeal mem- } \\
\text { brane oxygenation }\end{array}$ & $2(0.4)$ & $0(0.0)$ & $0(0.0)$ & $1(0.7)$ & $1(1.0)$ & 0.4553 & 0.0507 \\
\hline \multirow[t]{9}{*}{ Medical drugs } & $\begin{array}{l}\text { Traditional Chinese } \\
\text { medicine }\end{array}$ & $79(15.9)$ & $8(5.6)$ & $14(11.4)$ & $27(20.0)$ & $30(31.3)$ & $<0.0001$ & $<0.0001$ \\
\hline & Immunoglobulin & $134(27.0)$ & $15(10.6)$ & $33(26.8)$ & $33(24.4)$ & $53(55.2)$ & $<0.0001$ & $<0.0001$ \\
\hline & Interferon & $387(78.0)$ & $117(82.4)$ & $94(76.4)$ & $99(73.3)$ & $77(80.2)$ & 0.2906 & 0.4539 \\
\hline & Antioxidants & $134(27.0)$ & $22(15.5)$ & $27(22.0)$ & $38(28.1)$ & $47(49.0)$ & $<0.0001$ & $<0.0001$ \\
\hline & Glucocorticoid & $123(24.8)$ & $12(8.5)$ & 19(15.4) & $41(30.4)$ & $51(53.1)$ & $<0.0001$ & $<.0001$ \\
\hline & Thymosin & $130(26.2)$ & $19(13.4)$ & $27(22.0)$ & $39(28.9)$ & $45(46.9)$ & $<0.0001$ & $<.0001$ \\
\hline & Neurotrophic drugs & $95(19.2)$ & $15(10.6)$ & 17(13.8) & $35(25.9)$ & $28(29.2)$ & 0.0002 & $<0.0001$ \\
\hline & Any antibiotics & $283(57.1)$ & $53(37.3)$ & $65(52.8)$ & $89(65.9)$ & $76(79.2)$ & $<0.0001$ & $<0.0001$ \\
\hline & Any antivirals & $471(95.0)$ & $135(95.1)$ & $116(94.3)$ & $128(94.8)$ & $92(95.8)$ & 0.9762 & 0.6806 \\
\hline
\end{tabular}

$* P$ value from testing differences in proportions among different pulmonary opacity groups

$\dagger P$ value from trend test of linear pulmonary opacity effect in generalized linear model in which pulmonary opacity (\%) was included as the only predictor

pulmonary opacity score and clinical features and outcomes in COVID-19 patients. The study demonstrated that demographic, epidemiological, clinical, laboratory features on admission, and clinical management during hospitalization showed a linear trend by the quartile CT pulmonary opacity score. CT score correlated with poor clinical outcomes including severe and critical illness, ICU admission, respiratory failure and prolonged hospital stay. Our study introduced a simple quantitative parameter of pulmonary opacity score from CT to describe lung involvement which is reproducible for radiologists.

To facilitate better communication between radiologists and physicians, different classification systems of COVID19 incorporating imaging findings [e.g., ground-glass opacities (GGO), consolidation, crazy-paving pattern and fibrosis] have been developed [14]. CT imaging findings play a role in the classification and staging of COVID-19, early detection in relation to a serological test, disease severity and guide to therapy, surveillance with the response to therapy, prediction of secondary bacterial infection, differentiation from simulating lesions, and screening with prevention and control [14]. Follow-up CT scans were used for longitudinal evaluation by allowing the detection of disease progression, complications, and suspected acute respiratory deteriorations [15]. Advanced imaging modalities, such as quantitative CT techniques may also be valuable in delineation of the pulmonary distribution (GGO and consolidations) to assess the disease severity and detect disease progression on follow-up $[14,15]$. To calculate the opacity extent, we employed a more precise CT scoring system that used the percentage of pulmonary opacity area relative to the entire lung zone on CT image in the unit of $5 \%$. Other studies applied crude CT scoring systems. For example, Wang et al. used 0:0\%; $1: 1-49 \%$; $2: 50-75 \%$; $3:>75 \%$; range $0-3$; global score $0-15$ [16]; Hu et al. used 0:0\%; 1:1-25\%; 2:26-50\%; 3:51-75\%; 4:76-100\%; range 0-4; global score 0-20 [17-21]; Francone et al. used $0: 0 \% ; 1:<5 \% ; 2: 5-25 \% ; 3: 26-50 \% ; 4: 51-75 \%$; 5: $>75 \%$; range 0-5; global score 0-25 [22, 23]; Zhao et al. 
Table 4 Factors associated with pulmonary opacity score in patients with COVID-19: Results from linear regression analysis

\begin{tabular}{|c|c|c|c|c|}
\hline \multirow[t]{2}{*}{ Variables } & \multicolumn{2}{|l|}{ Univariate analysis } & \multirow{2}{*}{$\begin{array}{l}\text { Multivariate analysis } \\
\text { Estimate }(95 \% \mathrm{CI})\end{array}$} & \multirow[t]{2}{*}{$P$ value } \\
\hline & Estimate $(95 \% \mathrm{CI})$ & $P$ value & & \\
\hline Male & $5.16(1.30,9.02)$ & 0.0088 & $1.20(-2.22,4.63)$ & 0.4917 \\
\hline Age (year) & $0.41(0.30,0.52)$ & $<0.0001$ & $0.29(0.19,0.39)$ & $<0.0001$ \\
\hline Single onset & $13.22(9.53,16.91)$ & $<0.0001$ & $10.29(6.86,13.72)$ & $<0.0001$ \\
\hline Fever & $12.27(8.26,16.29)$ & $<0.0001$ & $4.67(0.89,8.46)$ & 0.0158 \\
\hline Cough & $8.29(4.46,12.13)$ & $<0.0001$ & $4.13(0.32,7.94)$ & 0.0340 \\
\hline Sputum & $8.88(4.54,13.21)$ & $<0.0001$ & $2.32(-1.96,6.59)$ & 0.2895 \\
\hline Hypertension & $11.62(6.19,17.06)$ & $<0.0001$ & $2.45(-2.65,7.55)$ & 0.3471 \\
\hline Diabetes & $14.02(6.12,21.92)$ & 0.0005 & $3.42(-3.73,10.57)$ & 0.3492 \\
\hline Smoker & $9.48(1.40,17.56)$ & 0.0215 & $4.14(-2.91,11.19)$ & 0.2506 \\
\hline Temperature $\left({ }^{\circ} \mathrm{C}\right)$ & $6.58(4.09,9.06)$ & $<0.0001$ & $3.42(1.06,5.79)$ & 0.0047 \\
\hline Heart rate (beat/minute) & $0.23(0.09,0.38)$ & 0.0014 & $0.03(-0.10,0.17)$ & 0.6166 \\
\hline Respiratory rate (breath/minute) & $1.54(0.77,2.30)$ & $<0.0001$ & $0.41(-0.29,1.11)$ & 0.2504 \\
\hline $\begin{array}{l}\text { Peripheral capillary oxygen saturation } \\
\left(\mathrm{SpO}_{2} \text {, per } 5 \%\right)\end{array}$ & $-15.23(-19.51,-10.94)$ & $<0.0001$ & $-10.44(-14.57,-6.32)$ & $<0.0001$ \\
\hline
\end{tabular}

CI confidence interval

The estimates were coefficients

used $0: 0 \% ; 1:<25 \% ; 2: 25-49 \% ; 3: 50-74 \% ; 4: \geq 75 \%$; range 0-4; global score 0-24 [24-26]; Aalinezhad et al. used another system $(0: 0 \% ; 1:<50 \% ; 2:>50 \%$; range $0-2$; global score 0-40) [27, 28]. Similarly, Guillo et al. used a scoring system of $0-10 \%, 11-25 \%, 26-50 \%, 51-75 \%$ and $76-100 \%$ ground-glass opacities and consolidation [29]. The ICC value for pulmonary opacity score from the two radiologists (YW and SJ) in our study was closed to one, indicating high inter-observer consistency and reliability. Due to the good agreement of pulmonary opacity score assessed by the two radiologists, the average of pulmonary opacity scores was used when discrepancies occurred.

The study found that age, single onset, initial symptoms, body temperature and $\mathrm{SpO}_{2}$ on admission in patients with COVID-19 were independent predictors of CT pulmonary opacity score, indicating more severe lung function injury. This is consistent with the previous studies reporting that clinical characteristics including patient age and coexisting condition, immune status, body temperature and exposure history may be related to $\mathrm{CT}$ imaging in patients with viral pneumonia including SARS and COVID-19 [2, 9, 30, 31]. In addition, Aalinezhad et al.'s study also showed a significant inverse relationship between CT severity score and $\mathrm{SpO}_{2}$ [28].

Our study showed that in patients with COVID-19, as CT pulmonary opacity score increased, platelet count and albumin level decreased, while the level of CRP and fibrinogen elevated, and $\geq 41 \%$ of lung involvement was associated with more severe lymphopenia, suggesting signs of viral infection and inflammation, abnormal coagulation function and liver function. Previous studies reported that CT severity showed a positive association with CRP level and negative correlation with lymphocyte count $[32,33]$. Francone et al. found the semi-quantitative CT score was significantly correlated with CRP (correlation coefficient $r=0.62$ ) and D-dimer $(r=0.66)$ levels [22], while our data provided some evidence to support the association with CRP but not with D-dimer. A study demonstrated that age and monocyte-lymphocyte ratio may predict imaging progression on chest CT in COVID-19 patients [34]. Other studies indicated moderate positive correlations between $\mathrm{CT}$ severity score and transferrin, lactate dehydrogenase, troponin, and inflammation-related factors of leucocytes, neutrophils, and IL-2R ( $r$ range $0.45-0.60)$ $[17,35]$, although we were unable to verify those correlations due to lack of data.

The study presented that the proportion of patients receiving medical treatment and oxygen support increased with the rise of pulmonary opacity score. Khosravi et al. also found patients with baseline CT severity score $>8$ had threefold higher risk of intubation [25]. Our data showed that pulmonary opacity score, especially when $\geq 41 \%$, may be an accurate indicator of severe or critical illness, acute respiratory failure and intensive care requirements. Zhao et al. evaluated lung involvement using another CT scoring system and also found mean score was higher in the emergency group (mild and common types) than in the nonemergency group (severe and fatal types) [24]. More studies showed the CT score calculated by their scoring systems was significantly higher in critical and severe than in mild and common category $[18,19,22]$. Other studies found that the overall lung involvement score had predictive value for clinical severity and ICU admission, and higher chest CT score 
Table 5 Effects of pulmonary opacity score on clinical outcomes in patients with COVID-19: Results from generalized linear model analysis

\begin{tabular}{|c|c|c|c|c|c|c|}
\hline \multirow[t]{2}{*}{ Clinical outcome ${ }^{* *}$} & \multirow[t]{2}{*}{ Pulmonary opacity score } & \multirow[t]{2}{*}{$\mathrm{n} / \mathrm{N}(\%)$ or $N$, Mean $(\mathrm{SD})$} & \multicolumn{2}{|l|}{ Unadjusted analysis } & \multicolumn{2}{|l|}{ Adjusted analysis* } \\
\hline & & & Estimate $(95 \% \mathrm{CI})$ & $P$ value & Estimate $(95 \% \mathrm{CI})$ & $P$ value \\
\hline \multirow[t]{6}{*}{$\begin{array}{l}\text { Severe or critically ill } \\
\text { (Yes or no) }\end{array}$} & $\begin{array}{l}\text { Score as continuous vari- } \\
\text { able (per 5\%) }\end{array}$ & $58 / 496(11.7 \%)$ & $1.38(1.28,1.49)$ & $<0.0001$ & $1.35(1.22,1.49)$ & $<0.0001$ \\
\hline & \multicolumn{6}{|l|}{ Score as categorical variable } \\
\hline & $\leq 5 \%$ & $3 / 142(2.1 \%)$ & 1.00 & & 1.00 & \\
\hline & $6-20 \%$ & $6 / 123(4.9 \%)$ & $2.38(0.58,9.71)$ & 0.2282 & $1.79(0.37,8.53)$ & 0.4665 \\
\hline & $21-40 \%$ & $11 / 135(8.1 \%)$ & $4.11(1.12,15.07)$ & 0.0330 & $2.19(0.50,9.61)$ & 0.2998 \\
\hline & $41 \%+$ & $38 / 96(39.6 \%)$ & $30.36(9.01,102.28)$ & $<0.0001$ & $15.58(3.82,63.53)$ & 0.0001 \\
\hline \multirow[t]{6}{*}{$\begin{array}{l}\text { ICU admission (Yes or } \\
\text { no) }\end{array}$} & $\begin{array}{l}\text { Score as continuous vari- } \\
\text { able (per } 5 \%)\end{array}$ & $64 / 496(12.9 \%)$ & $1.31(1.22,1.39)$ & $<0.0001$ & $1.27(1.16,1.38)$ & $<0.0001$ \\
\hline & \multicolumn{6}{|l|}{ Score as categorical variable } \\
\hline & $\leq 5 \%$ & $7 / 142(4.9 \%)$ & 1.00 & & 1.00 & \\
\hline & $6-20 \%$ & $7 / 123(5.7 \%)$ & $1.16(0.40,3.42)$ & 0.7824 & $0.77(0.22,2.62)$ & 0.6713 \\
\hline & $21-40 \%$ & $12 / 135(8.9 \%)$ & $1.88(0.72,4.93)$ & 0.1986 & $0.91(0.29,2.86)$ & 0.8700 \\
\hline & $41 \%+$ & $38 / 96(39.6 \%)$ & $12.64(5.33,29.95)$ & $<0.0001$ & $6.26(2.15,18.23)$ & 0.0008 \\
\hline \multirow[t]{6}{*}{$\begin{array}{l}\text { Respiratory failure (Yes } \\
\text { or no) }\end{array}$} & $\begin{array}{l}\text { Score as continuous vari- } \\
\text { able (per 5\%) }\end{array}$ & $55 / 496(11.1 \%)$ & $1.39(1.28,1.50)$ & $<0.0001$ & $1.39(1.25,1.55)$ & $<0.0001$ \\
\hline & \multicolumn{6}{|l|}{ Score as categorical variable } \\
\hline & $\leq 5 \%$ & $3 / 142(2.1 \%)$ & 1.00 & & 1.00 & \\
\hline & $6-20 \%$ & $5 / 123(4.1 \%)$ & $1.96(0.46,8.39)$ & 0.3626 & $1.66(0.32,8.54)$ & 0.5419 \\
\hline & $21-40 \%$ & $10 / 135(7.4 \%)$ & $3.71(1.00,13.77)$ & 0.0504 & $2.37(0.52,10.90)$ & 0.2658 \\
\hline & $41 \%+$ & $37 / 96(38.5 \%)$ & $29.06(8.62,97.96)$ & $<0.0001$ & $19.49(4.55,83.40)$ & $<0.0001$ \\
\hline \multirow[t]{6}{*}{$\begin{array}{l}\text { Hospital stay (Continuous } \\
\text { variable, day) }\end{array}$} & $\begin{array}{l}\text { Score as continuous vari- } \\
\text { able (per 5\%) }\end{array}$ & $496,17.62(7.53)$ & $0.32(0.17,0.47)$ & $<0.0001$ & $0.24(0.07,0.41)$ & 0.0047 \\
\hline & \multicolumn{6}{|l|}{ Score as categorical variable } \\
\hline & $\leq 5 \%$ & $142,15.94(6.36)$ & 0.00 & & 0.00 & \\
\hline & $6-20 \%$ & $123,17.36(7.45)$ & $1.42(-0.37,3.21)$ & 0.1192 & $1.13(-0.65,2.91)$ & 0.2148 \\
\hline & $21-40 \%$ & $135,18.20(7.84)$ & $2.26(0.52,4.01)$ & 0.0110 & $1.37(-0.45,3.18)$ & 0.1397 \\
\hline & $41 \%+$ & $96,19.65(8.27)$ & $3.71(1.79,5.63)$ & 0.0002 & $2.59(0.46,4.72)$ & 0.0170 \\
\hline
\end{tabular}

${ }^{* * *}$ For severe or critically ill, intensive care unit admission and respiratory failure, the estimates were odds ratios; while for hospital stay, the estimates were coefficients

$S D$ standard deviation, $I C U$ intensive care unit

*All variables in Table 1 were included in the adjusted analysis

was significantly associated with an increase in requirement of oxygen and even mechanical ventilation [16, 25-27, 36, 37]. CT involvement score can help early diagnosis, severity assessment and treatment of COVID-19 [9, 38, 39] and may indicate the progression and recovery of the disease $[6,40]$. These findings are similar to that of SARS that the extent of lung opacification was an objective prognostic indicator of death, disease severity and requirements of aggressive therapy for assisted ventilation or oxygen supplementation [41-43], and pulmonary opacity resolved over time in convalescent patients $[44,45]$. We found patients with more pulmonary opacity stayed longer in hospital. This may be due to more severe illness and more medical treatment and oxygen support associated with higher pulmonary opacity score. Previous evidence suggested that patients having bilateral pneumonia were hospitalized longer than those with normal CT scan results [12] and patients with higher CT scores may have more prolonged disease course and hospital stay [21, 46, 47]. Our study did not assess the association between pulmonary opacity score and mortality because of no recorded death in the study sample as a result of early recognition and intervention [48].

The study had several merits compared with previous studies. First, all eligible COVID-19 patients with and without symptoms, including asymptomatic, mild, moderate, severe and critically ill, in Jiangsu province, were included in the current study whereas most of the previous studies focused on the patients with moderate, severe or critically ill symptoms. Therefore, results from this study are more generalizable to the COVID-19 patients with a wide spectrum of infections, particularly in the post-COVID-19 era when severe cases are supposed to decrease. Second, the study 
provided some novel and robust information on associations between pulmonary opacity score and demographic, epidemiological, clinical, laboratory features and clinical management based on a larger dataset $(N=496)$ than most previous studies on the similar topic (mostly $N=50-200$ ). Third, the present study assessed impacts of pulmonary opacity score at admission on a number of important clinical outcomes (including disease severity, ICU admission, respiratory failure, and hospital stay), instead of a single clinical outcome alone in most previous reports, thus providing a more comprehensive perspective of the relationships between pulmonary opacity score and various clinical outcomes in patients with COVID-19. Fourth, to assess effects of pulmonary opacity score on clinical outcomes, the pulmonary opacity score was measured in the unit of 5\% and treated as both continuous and categorical variables in the generalized linear models, and the results were robust regardless of CT score function forms. This is in contrast to most previous studies in which CT score was treated as either a continuous variable or binary variable, which may have generated biased statistical results due to possibly wrong specification of CT score.

This study had some limitations. First, the study excluded 129 patients who did not take CT but X-ray examination and hence had no CT pulmonary opacity score, so selection bias may occur. Second, although this study analyzed the effects of pulmonary opacity scores at admission on disease severity, ICU admission and respiratory failure during the whole study period, we only recorded CT at the time of admission; hence it was impossible to analyze the change in pulmonary opacity scores throughout the study period. Third, due to lack of data, we did not analyze associations between CT pulmonary opacity score and some other characteristics, e.g., transferrin, leucocytes and platelet-lymphocyte ratios, which had been reported to be associated with pulmonary opacity score in the previous studies [17, 35, 49]. For the same reason, we did not analyze the association between CT findings and pulmonary embolism although a previous study has showed a high pulmonary embolism prevalence at CT pulmonary angiography in patients testing positive for COVID-19 [50]. Lastly, this was an observational study and the observed results may still be subject to possible unobserved confounding factors.

\section{Conclusion}

The degree of CT pulmonary opacity was closely related to age, single onset, fever, cough, $\mathrm{SpO}_{2}$, lymphocyte count, platelet count, albumin level, CRP level and fibrinogen level. Patients with high pulmonary opacity score, in particular, $\geq 41 \%$, had a high risk of severe or critical illness, ICU admission, respiratory failure and long hospital stay.
Author contributions HL: Conceptualization, Formal analysis, Methodology, Writing — original draft. YW: Resources, Data Curation, Investigation, Writing - review and editing. SL: Resources, Data curation, Investigation, Writing - review and editing. RC: Methodology, Writing-review and editing. TC: Methodology, Writing—review and editing. YY: Resources, Data curation, Investigation. DW: Conceptualization, Software, Formal analysis, Supervision, Writing-review and editing. SJ: Conceptualization, Supervision, Validation, Writingreview and editing.

Funding This work was supported, in part, by the research Grant 2020YFC0843700 67 from Ministry of Science and Technology of the People's Republic of China. The funding source had no involvement in study design; in the collection, analysis and interpretation of data; in the writing of the report; or in the decision to submit the article for publication.

Data availability The datasets used and/or analyzed during the current study are available from the corresponding author on reasonable request.

\section{Declarations}

Conflict of interest The authors declare that they have no conflict of interest.

Informed consent statement Patient informed consent was waived due to the retrospective study design.

\section{References}

1. Jardon M, Mohammad SF, Jude CM, Pahwa A (2019) Imaging of emerging infectious diseases. Curr Radiol Rep 7(9):25

2. Koo HJ, Lim S, Choe J, Choi SH, Sung H, Do KH (2018) Radiographic and CT features of viral pneumonia. Radiographics 38(3):719-739

3. Cao Y, Liu X, Xiong L, Cai K (2020) Imaging and clinical features of patients with 2019 novel coronavirus SARS-CoV-2: a systematic review and meta-analysis. J Med Virol 92(9):1449-1459

4. Long C, Xu H, Shen Q, Zhang X, Fan B, Wang C, Zeng B, Li Z, Li X, Li H (2020) Diagnosis of the coronavirus disease (COVID19): rRT-PCR or CT? Eur J Radiol. https://doi.org/10.1016/j.ejrad. 2020.108961

5. Caruso D, Zerunian M, Polici M, Pucciarelli F, Polidori T, Rucci C, Guido G, Bracci B, de Dominicis C, Laghi A (2020) Chest CT features of COVID-19 in Rome, Italy. Radiology 296(2):E79-E85

6. Ye Z, Zhang Y, Wang Y, Huang Z, Song B (2020) Chest CT manifestations of new coronavirus disease 2019 (COVID-19): a pictorial review. Eur Radiol 30(8):4381-4389

7. Dai H, Zhang X, Xia J, Zhang T, Shang Y, Huang R, Liu R, Wang D, Li M, Wu J et al (2020) High-resolution chest CT features and clinical characteristics of patients infected with COVID-19 in Jiangsu, China. Int J Infect Dis 95:106-112

8. Wu J, Wu X, Zeng W, Guo D, Fang Z, Chen L, Huang H, Li C (2020) Chest CT findings in patients with coronavirus disease 2019 and its relationship with clinical features. Invest Radiol 55(5):257-261

9. Zhang X, Cai H, Hu J, Lian J, Gu J, Zhang S, Ye C, Lu Y, Jin $\mathrm{C}$, Yu G et al (2020) Epidemiological, clinical characteristics of cases of SARS-CoV-2 infection with abnormal imaging findings. Int J Infect Dis 94:81-87 
10. Noll E, Soler L, Ohana M, Ludes PO, Pottecher J, Bennett-Guerrero E, Veillon F, Goichot B, Schneider F, Meyer N et al (2020) A novel, automated, quantification of abnormal lung parenchyma in patients with COVID-19 infection: initial description of feasibility and association with clinical outcome. Anaesth Crit Care Pain Med. https://doi.org/10.1016/j.accpm.2020.10. 014

11. Cozzi D, Albanesi M, Cavigli E, Moroni C, Bindi A, Luvarà S, Lucarini S, Busoni S, Mazzoni LN, Miele V (2020) Chest X-ray in new coronavirus disease 2019 (covid-19) infection: findings and correlation with clinical outcome. Radiol Med 125(8):730-737

12. Wu S, Xue L, Legido-Quigley H, Khan M, Wu H, Peng X, Li X, Li P (2020) Understanding factors influencing the length of hospital stay among non-severe COVID-19 patients: a retrospective cohort study in a Fangcang shelter hospital. PLoS ONE. https:// doi.org/10.1371/journal.pone.0240959

13. National Health Commission and National Administration of Traditional Chinese Medicine (2020) Diagnosis and treatment protocol for novel coronavirus pneumonia (trial version 7). Chin Med J (Engl) 133(9):1087-1095

14. Razek A, Fouda N, Fahmy D, Tanatawy MS, Sultan A, Bilal M, Zaki M, Abdel-Aziz M, Sobh D (2021) Computed tomography of the chest in patients with COVID-19: what do radiologists want to know? Pol J Radiol 86:e122-e135

15. Razek A, El Badrawy MK, Alnaghy E (2020) Interstitial lung fibrosis imaging reporting and data system: what radiologist wants to know? J Comput Assist Tomogr 44(5):656-666

16. Wang X, Hu X, Tan W, Mazzone P, Mireles-Cabodevila E, Han XZ, Huang P, Hu W, Dweik R, Cheng Z (2020) Multi-center study of temporal changes and prognostic value of a CT visual severity score in hospitalized patients with COVID-19. AJR Am J Roentgenol. https://doi.org/10.2214/AJR.20.24044

17. Hu Y, Zhan C, Chen C, Ai T, Xia L (2020) Chest CT findings related to mortality of patients with COVID-19: a retrospective case-series study. PLoS ONE. https://doi.org/10.1371/journal. pone. 0237302

18. Li K, Fang Y, Li W, Pan C, Qin P, Zhong Y, Liu X, Huang M, Liao Y, Li S (2020) CT image visual quantitative evaluation and clinical classification of coronavirus disease (COVID-19). Eur Radiol 30(8):4407-4416

19. Liu N, He G, Yang X, Chen J, Wu J, Ma M, Lu W, Li Q, Cheng $\mathrm{T}$, Huang X (2020) Dynamic changes of chest CT follow-up in coronavirus disease-19 (COVID-19) pneumonia: relationship to clinical typing. BMC Med Imaging 20(1):92

20. Tabatabaei SMH, Rahimi H, Moghaddas F, Rajebi H (2020) Predictive value of $\mathrm{CT}$ in the short-term mortality of coronavirus disease 2019 (COVID-19) pneumonia in nonelderly patients: a case-control study. Eur J Radiol. https://doi.org/10.1016/j.ejrad. 2020.109298

21. Zhan J, Li H, Yu H, Liu X, Zeng X, Peng D, Zhang W (2021) 2019 novel coronavirus (COVID-19) pneumonia: CT manifestations and pattern of evolution in 110 patients in Jiangxi, China. Eur Radiol 31(2):1059-1068

22. Francone M, Iafrate F, Masci GM, Coco S, Cilia F, Manganaro L, Panebianco V, Andreoli C, Colaiacomo MC, Zingaropoli MA et al (2020) Chest CT score in COVID-19 patients: correlation with disease severity and short-term prognosis. Eur Radiol 30(12):6808-6817

23. Pan F, Ye T, Sun P, Gui S, Liang B, Li L, Zheng D, Wang J, Hesketh RL, Yang L et al (2020) Time course of lung changes at chest CT during recovery from coronavirus disease 2019 (COVID-19). Radiology 295(3):715-721

24. Zhao W, Zhong Z, Xie X, Yu Q, Liu J (2020) Relation between chest $\mathrm{CT}$ findings and clinical conditions of coronavirus disease (COVID-19) pneumonia: a multicenter study. AJR Am J Roentgenol 214(5):1072-1077
25. Khosravi B, Aghaghazvini L, Sorouri M, Naybandi Atashi S, Abdollahi M, Mojtabavi H, Khodabakhshi M, Motamedi F, Azizi F, Rajabi Z et al (2021) Predictive value of initial CT scan for various adverse outcomes in patients with COVID-19 pneumonia. Heart Lung 50(1):13-20

26. Li S, Liu S, Wang B, Li Q, Zhang H, Zeng L, Ge H, Ma Q, Shen N (2021) Predictive value of chest CT scoring in COVID19 patients in Wuhan, China: a retrospective cohort study. Respir Med. https://doi.org/10.1016/j.rmed.2020.106271

27. Yang R, Li X, Liu H, Zhen Y, Zhang X, Xiong Q, Luo Y, Gao C, Zeng W (2020) Chest CT severity score: an imaging tool for assessing severe COVID-19. Radiol Cardiothorac Imaging 2(2):e200047

28. Aalinezhad M, Alikhani F, Akbari P, Rezaei MH, Soleimani S, Hakamifard A (2021) Relationship between CT severity score and capillary blood oxygen saturation in patients with COVID-19 infection. Indian J Crit Care Med 25(3):279-283

29. Guillo E, Bedmar Gomez I, Dangeard S, Bennani S, Saab I, Tordjman M, Jilet L, Chassagnon G, Revel MP (2020) COVID-19 pneumonia: diagnostic and prognostic role of CT based on a retrospective analysis of 214 consecutive patients from Paris, France. Eur J Radiol. https://doi.org/10.1016/j.ejrad.2020.109209

30. Paul NS, Chung T, Konen E, Roberts HC, Rao TN, Gold WL, Mehta S, Tomlinson GA, Boylan CE, Grossman H et al (2004) Prognostic significance of the radiographic pattern of disease in patients with severe acute respiratory syndrome. AJR Am J Roentgenol 182(2):493-498

31. Chen Z, Fan H, Cai J, Li Y, Wu B, Hou Y, Xu S, Zhou F, Liu Y, Xuan W et al (2020) High-resolution computed tomography manifestations of COVID-19 infections in patients of different ages. Eur J Radiol. https://doi.org/10.1016/j.ejrad.2020.108972

32. Wang K, Kang S, Tian R, Zhang X, Zhang X, Wang Y (2020) Imaging manifestations and diagnostic value of chest $\mathrm{CT}$ of coronavirus disease 2019 (COVID-19) in the Xiaogan area. Clin Radiol 75(5):341-347

33. Tan C, Huang Y, Shi F, Tan K, Ma Q, Chen Y, Jiang X, Li X (2020) C-reactive protein correlates with computed tomographic findings and predicts severe COVID-19 early. J Med Virol 92(7):856-862

34. Yang Z, Shi J, He Z, Lu Y, Xu Q, Ye C, Chen S, Tang B, Yin K, Lu Y et al (2020) Predictors for imaging progression on chest CT from coronavirus disease 2019 (COVID-19) patients. Aging (Albany NY) 12(7):6037-6048

35. Azab SM, Zytoon AA, Kasemy ZAA, Omar SF, Ewida SF, Sakr KA, Ella TFA (2021) Learning from pathophysiological aspects of COVID-19 clinical, laboratory, and high-resolution CT features: a retrospective analysis of 128 cases by disease severity. Emerg Radiol. https://doi.org/10.1007/s10140-020-01875-1

36. Kodama T, Obinata H, Mori H, Murakami W, Suyama Y, Sasaki H, Kouzaki Y, Kawano S, Kawana A, Mimura S (2021) Prediction of an increase in oxygen requirement of SARS-CoV-2 pneumonia using three different scoring systems. J Infect Chemother 27(2):336-341

37. Liu S, Nie C, Xu Q, Xie H, Wang M, Yu C, Hou X (2021) Prognostic value of initial chest $\mathrm{CT}$ findings for clinical outcomes in patients with COVID-19. Int J Med Sci 18(1):270-275

38. Xu YH, Dong JH, An WM, Lv XY, Yin XP, Zhang JZ, Dong L, Ma X, Zhang HJ, Gao BL (2020) Clinical and computed tomographic imaging features of novel coronavirus pneumonia caused by SARS-CoV-2. J Infect 80(4):394-400

39. Liu KC, Xu P, Lv WF, Qiu XH, Yao JL, Gu JF, Wei W (2020) CT manifestations of coronavirus disease-2019: a retrospective analysis of 73 cases by disease severity. Eur J Radiol. https://doi. org/10.1016/j.ejrad.2020.108941

40. Wang Y, Dong C, Hu Y, Li C, Ren Q, Zhang X, Shi H, Zhou $M$ (2020) Temporal changes of CT findings in 90 patients 
with COVID-19 pneumonia: a longitudinal study. Radiology 296(2):E55-E64

41. Antonio GE, Ooi CG, Wong KT, Tsui EL, Wong JS, Sy AN, Hui JY, Chan CY, Huang HY, Chan YF et al (2005) Radiographicclinical correlation in severe acute respiratory syndrome: study of 1373 patients in Hong Kong. Radiology 237(3):1081-1090

42. Chau TN, Lee PO, Choi KW, Lee CM, Ma KF, Tsang TY, Tso YK, Chiu MC, Tong WL, Yu WC et al (2004) Value of initial chest radiographs for predicting clinical outcomes in patients with severe acute respiratory syndrome. Am J Med 117(4):249-254

43. Ooi CG, Khong PL, Ho JC, Lam B, Wong WM, Yiu WC, Wong PC, Wong CF, Lai KN, Tsang KW (2003) Severe acute respiratory syndrome: radiographic evaluation and clinical outcome measures. Radiology 229(2):500-506

44. Chang YC, Yu CJ, Chang SC, Galvin JR, Liu HM, Hsiao CH, Kuo PH, Chen KY, Franks TJ, Huang KM et al (2005) Pulmonary sequelae in convalescent patients after severe acute respiratory syndrome: evaluation with thin-section CT. Radiology 236(3):1067-1075

45. Hsu HH, Tzao C, Wu CP, Chang WC, Tsai CL, Tung HJ, Chen CY (2004) Correlation of high-resolution CT, symptoms, and pulmonary function in patients during recovery from severe acute respiratory syndrome. Chest 126(1):149-158

46. Cai Y, Liu J, Yang H, Chen T, Yu Q, Chen J, Huang D, Chen Z, Shang QL, Ma C et al (2021) Correlation between early features and prognosis of symptomatic COVID-19 discharged patients in Hunan, China. Sci Rep 11(1):4304

47. Kaya F, Konya P, Demirel E, Demirtürk N, Orhan S, Ufuk F (2021) Visual and quantitative assessment of COVID-19 pneumonia on chest CT: the relationship with disease severity and clinical findings. Curr Med Imaging. https://doi.org/10.2174/1573405617 666210215142528

48. Sun Q, Qiu H, Huang M, Yang Y (2020) Lower mortality of COVID-19 by early recognition and intervention: experience from Jiangsu Province. Ann Intensive Care 10(1):33

49. Çakmak V, Yilmaz A, Sari T, Çakmak P, Özen M, Herek D, Oskay A (2021) An evaluation of chest computed tomography and hemogram data in patients with COVID-19: the importance of the thymus. Turk J Med Sci. https://doi.org/10.3906/sag-2007-306

50. Kaminetzky M, Moore W, Fansiwala K, Babb JS, Kaminetzky D, Horwitz LI, McGuinness G, Knoll A, Ko JP (2020) Pulmonary embolism at CT pulmonary angiography in patients with COVID19. Radiol Cardiothorac Imaging. https://doi.org/10.1148/ryct. 2020200308

Publisher's Note Springer Nature remains neutral with regard to jurisdictional claims in published maps and institutional affiliations. 